\title{
Effects of Verbally Labeled Anchor Points on the Distributional Parameters of Rating Measures
}

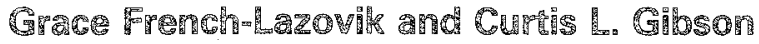 \\ Un:
}

The hypothesis was examined that the negative skew found in most distributions of performance ratings is a function of the verbal labels used as anchors. When verbal labels quantified on the basis of the range of real-life performance were employed, distributional parameters (means, skewness) were affected. Typically used sets of labels were shown to be more negative than believed, thus tending to force responses toward the high end of the scale and thereby contributing to negative skew.

The use of rating scales to obtain measures of performance has substantially increased in recent decades in basic research investigations and especially in applied evaluation research. Despite the large amount of research on rating methodology, some of the measurement parameters of these scales are not well understood. (Exhaustive reviews of this literature are provided by Wherry, 1950, for research published prior to 1950 , and by Landy \& Farr, 1980, for studies published between 1950 and 1978.) In particular, the factors that might bring some degree of control over the distributional parameters of rating scales have received little attention.

Typically, rating scalles provide a specified number of categories, usually representing a contin-

APPLIED PSYCHOLOGICAL MEASUREMENT

Vol. 8, No. 1, Winter 1984, pp. 49-57

(C) Copyright 1984 Applied Psychological Measurement Inc. 0146-6216/841010049-09\$1.70 uum, so that a respondent may choose one of the categories to express a judgment about some characteristic of an object or of human behavior. The term "rating scale" is generic and refers to a variety of methodologies for obtaining judgments, including the Semantic Differential (Osgood, 1952), the Q-sort (Stephenson, 1953), and the commonly used numerical rating scales. The principal ways in which these methods differ are in the number of anchor points (or defined categories) along the scale and the way in which the anchor points are described. Some of the most frequently used descriptors are verbal labels, marked lines between endpoints, integers, or integers in conjunction with verbal descriptors. It is commonly believed that numerical rating scales employing both integers and verbal descriptors provide the best aid to raters in defining scale positions.

Historically, with almost all rating scales, negatively skewed distributions of obtained ratings have resulted when human beings judge the overt behaviors of other humans in evaluative terms. The principal explanation in psychometric literature of this negative skew is the so-called "error of leniency" (Guilford, 1936), which refers to an assumed tendency on the part of a rater to be lenient in his or her ratings of other people. Postulating that other factors may contribute to the negative skew phenomenon, this research investigated the hypothesis that the distributional shape obtained 
from rating scales is dependent, in part, upon the choice of verbal labels used to anchor rating scale points.

A very commonly used evaluative rating scale in the behavioral sciences, and in industry as well, employs the following set of verbal anchorings: Very Above Average, Average, Below Average, Very Even those formats that differ from this one often have the label "average" as the midpoint of the scale. However, when ratings of human behaviors are made, the term "average" may be perjorative; people typically do not like to be described by this term. Thus, average may not be a true midpoint on a scale. Using a verbal label that is not a midpoint to anchor the middle category essentially forces the higher categories to be used more frequently than the middle. Consequently, the distribution of obtained scores is shifted higher on the scale, and a negatively skewed distribution is generated.

Investigating this problem necessitated bringing some objectivity to the choice of verbal descriptors. Other researchers have chosen labels by selecting words that have been psychophysically scaled as to the degree of favorableness they represent. An alternative approach, one more analogous to procedures used in test construction, would be the representation of a continuum based on the words that raters typically apply to the performance they are judging (in this instance by teachers) rather than on the continum indicated by psychophysically scaled anchor words. An empirical procedure was developed to derive numerical values, called descriptor indices, that locate the position of each of a set of possible verbal labels on a continuum by determining the proportion of teachers that potential raters would characterize by each label. It is hypothesized that these descriptor indices would function in a manner somewhat similar to item difficulty levels in test construction. By choosing verbal labels to anchor scale points on the basis of descriptor indices, two different rating scales were constructed and the relationship between their distributional parameters investigated. Evaluations of teaching made by college students provided the testing ground for this research.
Study I-Descriptor Indices

\section{Method}

Instrument. A set of 37 verbal labels culled from student evaluation of teaching rating scales was chosen in such a way as to ensure that the entire range of the continuum was spanned and the words were adequately familiar to college students (Thorndike \& Lorge, 1944). The instrument for obtaining data necessary to compute descriptor indices consisted of instructions, four examples of the task, and two sections presenting the same verbal labels, in two different random orders, to which subjects responded. In Section I each subject indicated the percentage of instructors in his or her high school or college career that he or she would characterize as falling (1) below and (2) at or above each of the verbal labels. Section II required subjects to respond, not from their own experience, but as they believed a typical student would respond. Section II was included to draw students' attention to the fact that their individual experiences might differ from those of a typical student. In particular, the term "average" was expected to be troublesome in that subjects might automatically indicate $50 \%$ falling above and below this point. Forcing subjects to attend to their own experiences was an attempt to hold such an effect to a minimum.

Subjects. Responses were obtained from 121 students taking courses in introductory psychology, statistics for psychology, and abnormal psychology.

Analyses. The percent indicated as falling below each label was pooled across subjects, and the mean percent and standard deviation were calculated separately for Sections I and II. On the basis of the mean percentages, verbal labels were rank ordered for each section and a Spearman rho computed to determine similarities in the two orderings. The standard deviation for each label was used as an indicator of the degree of agreement among respondents as to its scale position; the mean percent falling below each label in Section I was designated its "descriptor index," and these indices 
were related to integer scale points by the following formula:

$$
\begin{aligned}
& d=100\left[1+\frac{p-n}{n-1}\right] \\
& \begin{aligned}
\text { where } d & =\text { descriptor index }, \\
p & =\text { a scale point, and } \\
n & =\text { number of scale points, } \\
& \text { i.e., } p=1,2,3, \ldots, n .
\end{aligned}
\end{aligned}
$$

\section{Results}

Table 1 presents the summary statistics on the scale positions of 37 verbal labels calculated separately for the two different instructional sets, i.e., from the individual's experience and the typical student's view. Very high agreement between the two orderings of labels is demonstrated by the high Spearman tho $(r=.989)$. From the SDs it can be seen that there is greater agreement among individuals about the positions on the continuum of very favorable or very unfavorable labels than for labels near the middle of the scale. However, agreement is greater for the label "average" than for most of the other labels near that point. A $t$ statistic, calculated to test whether the descriptor index for "average" was significantly different from the theoretical midpoint of $50 \%$ gave the value $t$ $(107)=-4.749, p<.005$. Thus, the descriptor index for the label "average" falls below the scale midpoint, as hypothesized.

Attention should also be drawn to the labels "above average" and "below average." In terms of the descriptor indices, the distance from "above average" to "average" is $21.77 \%$, which is approximately equal to the distance from "average" to "below average" (18.74\%). This set of verbal labels characterizes a scale whose points are roughly equidistant, but the entire set is displaced toward the unfavorable end of the continuum, that is, not centered about the midpoint.

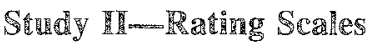

\section{Method}

Instruments. Two teacher rating questionnaires were constructed using identical item stems and instructions; they differed only in the labels attached to scale points. Five-point numerical scales on which each point was labeled with both an integer and a verbal descriptor were attached to a global rating item and to one item representing each of the three factors that predict the global one (French-Lazovik, 1974). The four items chosen were (1) Explanations were clear and understandable, (2) Material was presented in an interesting manner, (3) Student's intellectual curiosity was stimulated, and (4) Overall teaching ability.

Using the descriptor indices obtained on the basis of the first phase of data collection, labels were chosen for the two questionnaires, as illustrated in Table 2. It can be seen by comparing the descriptor indices (rounded to two digits) for the two sets of labels that those for the second questionnaire $\left(Q_{2}\right)$ are approximately one scale point lower than those for the first questionnaire $\left(Q_{1}\right)$. Thus, if descriptor indices do, in fact, act in a way similar to difficulty levels in test construction, then the mean numerical rating on $Q_{1}$ should be larger than on $Q_{2}$ for each item rated.

Subjects. Ratings on the four items were made by 304 students in the classes of six different instructors.

Procedure. Instructors were not present in their classes while the questionnaires were administered by a special proctor during the first 15 minutes of a class hour. Following instructions that guaranteed student anonymity, the two questionnaires were distributed in such a manner that half the students within a class randomly received $Q_{1}$, and half received $\mathrm{Q}_{2}$.

Data analysis. The question, "Do the verbal labels used to anchor the scale points of rating scales influence the ratings given?" was answered through separate analyses of variance for each item stem, using a randomized block design (Table 3 ). Classes were used as blocks in order to control for between-instructor variability.

\section{Results}

Table 4 contains the paired $t$ tests for assessing differences in the mean ratings resulting from the 
Table 1

Summary Statistics of the Proportion of Instructors Characterized As Falling Below Each Verbal Label

\begin{tabular}{|c|c|c|c|c|c|c|c|c|}
\hline \multirow[b]{2}{*}{$\begin{array}{l}\text { Verbal } \\
\text { Label }\end{array}$} & \multicolumn{4}{|c|}{ Section I } & \multicolumn{4}{|c|}{ Section II } \\
\hline & $\begin{array}{l}\text { Mean \%: } \\
\text { Descriptor } \\
\text { Index }\end{array}$ & SD & N & $\begin{array}{l}\text { Rank } \\
\text { Order }\end{array}$ & Mean \% & SD & $\mathbb{N}$ & $\begin{array}{l}\text { Rank } \\
\text { Order }\end{array}$ \\
\hline Exceptiona T & 91.311 & 8.707 & 119 & 1 & 92.378 & 6.263 & 111 & 1 \\
\hline Superior & 90.884 & 6.844 & 121 & 2 & 91.298 & 6.522 & 114 & 3 \\
\hline Outstanding & 90.681 & 7.167 & 119 & 3 & 91.578 & 6.660 & 116 & 2 \\
\hline Excellent & 90.432 & 8.675 & 118 & 4 & 90.848 & 7.180 & 112 & 4 \\
\hline Very High & 83.786 & 10.564 & 117 & 5 & 86.947 & 11.215 & 114 & 5 \\
\hline Very Good & 79.217 & 10.922 & 120 & 6 & 79.862 & 10.477 & 109 & 9 \\
\hline High & 79.147 & 10.669 & 116 & 7 & 82.574 & 10.408 & 115 & 6 \\
\hline Very Competent & 76.860 & 15.099 & 107 & 8 & 77.466 & 19.361 & 116 & 10 \\
\hline Very Effective & 76.783 & 12.685 & 115 & 9 & 82.514 & 11.060 & 111 & 7 \\
\hline $\begin{array}{c}\text { Well Above the } \\
\text { Average }\end{array}$ & 75.853 & 12.544 & 116 & 10 & 81.561 & 10.867 & 107 & 8 \\
\hline Better than Most & 74.919 & 15.687 & 111 & 11 & 70.835 & 17.147 & 109 & 11 \\
\hline Above Average & 66.923 & 14.884 & 117 & 12 & 67.512 & 16.896 & 121 & 12 \\
\hline Fine & 61.407 & 19.189 & 108 & 13 & 56.061 & 19.985 & 115 & 15 \\
\hline Effective & 57.875 & 15.748 & 104 & 14 & 56.588 & 20.033 & 114 & 14 \\
\hline Good & 56.514 & 13.600 & 107 & 15 & 58.845 & 16.225 & 116 & 13 \\
\hline Moderately Good & 54.520 & 15.166 & 102 & 16 & 52.692 & 17.676 & 120 & 16 \\
\hline $\begin{array}{c}\text { Compares Well with } \\
\text { the Average }\end{array}$ & h 53.705 & 15.587 & 105 & 17 & 51.233 & 17.085 & 120 & 18 \\
\hline Middling & 47.374 & 10.413 & 115 & 18 & 45.809 & 11.161 & 115 & 23 \\
\hline Competent & 47.000 & 20.714 & 119 & 19 & 48.488 & 20.013 & 121 & 20 \\
\hline Satisfactory & 46.602 & 17.045 & 113 & 20 & 51.446 & 20.287 & 121 & 17 \\
\hline Needs Improvement & 45.880 & 30.642 & 117 & 21 & 51.059 & 28.833 & 118 & 19 \\
\hline Average & 45.157 & 10.606 & 108 & 22 & 47.383 & 11.341 & 115 & 21 \\
\hline Adequate & 40.441 & 16.669 & 111 & 23 & 46.458 & 17.718 & 120 & 22 \\
\hline Mediocre & 38.926 & 13.866 & 121 & 24 & 41.906 & 14.791 & 117 & 24 \\
\hline Only Fair & 32.212 & 12.570 & 104 & 25 & 38.248 & 14.076 & 117 & 25 \\
\hline Below Average & 26.419 & 12.519 & 114 & 26 & 30.791 & 13.303 & 110 & 26 \\
\hline Unsatisfactory & 19.692 & 12.394 & 117 & 27 & 25.481 & 12.396 & 104 & 27 \\
\hline Ineffective & 19.336 & 11.769 & 116 & 28 & 22.730 & 12.613 & 115 & 28 \\
\hline Weak & 18.782 & 11.572 & 119 & 29 & 21.770 & 11.711 & 113 & 29 \\
\hline Low & 18.308 & 10.389 & 120 & 30 & 16.113 & 10.578 & 115 & 33 \\
\hline Ranks Below Most & 17.802 & 13.860 & 111 & 31 & 19.942 & 12.661 & 103 & 30 \\
\hline Inadequate & 17.188 & 11.434 & 117 & 32 & 18.384 & 11.429 & 112 & 31 \\
\hline Poor & 15.250 & 10.386 & 120 & 33 & 16.664 & 9.937 & 112 & 32 \\
\hline Inferior & 14.529 & 9.651 & 119 & 34 & 14.179 & 9.315 & 112 & 36 \\
\hline Very Low & 13.442 & 9.068 & 120 & 35 & 15.441 & 8.919 & 111 & 34 \\
\hline Very Ineffective & 12.356 & 9.532 & 118 & 36 & 14.708 & 10.004 & 106 & 35 \\
\hline Very Poor & 11.033 & 8.517 & 120 & 37 & 11.640 & 8.293 & 114 & 37 \\
\hline
\end{tabular}

two questionnaires. Additionally, when the individual class means are compared (Table 5 ), $Q_{1}$ is larger than $\mathrm{Q}_{2}$, as hypothesized, in 21 of the 24 possible comparisons (four items in each of six classes). The three deviations from this hypothesis all occurred in the same class, and the authors believe that an event that took place while the rat- ings were being made contributed to carelessness on the part of the students. (The instructor, waiting outside the classroom, posted grades from a recent exam; students hurriedly finished the ratings in order to go out and see their grades). Clearly, the mean ratings from $Q_{1}$ and $Q_{2}$ are different, and in the hypothesized direction. 
Table 2

Verbal Labels and Their Descriptor Indices (DI) for Questionnaires 1 and 2

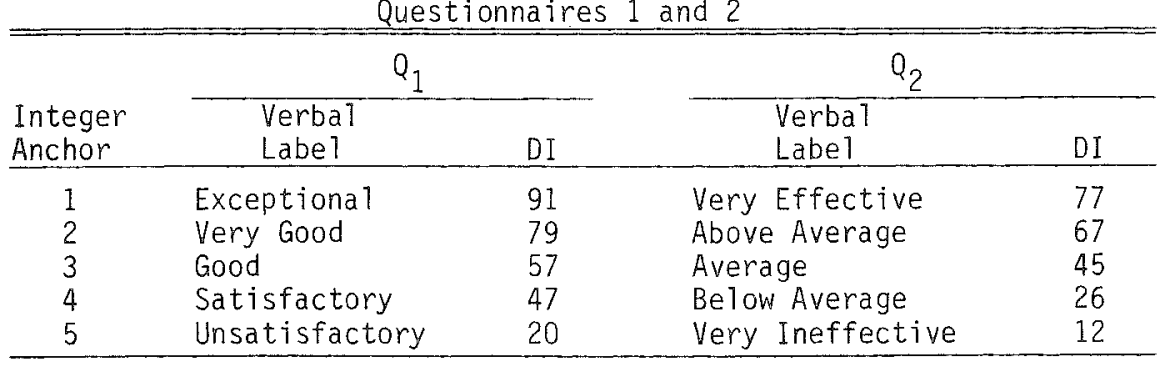

Table 3

Randomized Block ANOVA of Differences in the Mean Ratings for the Two Teacher Rating Questionnaires

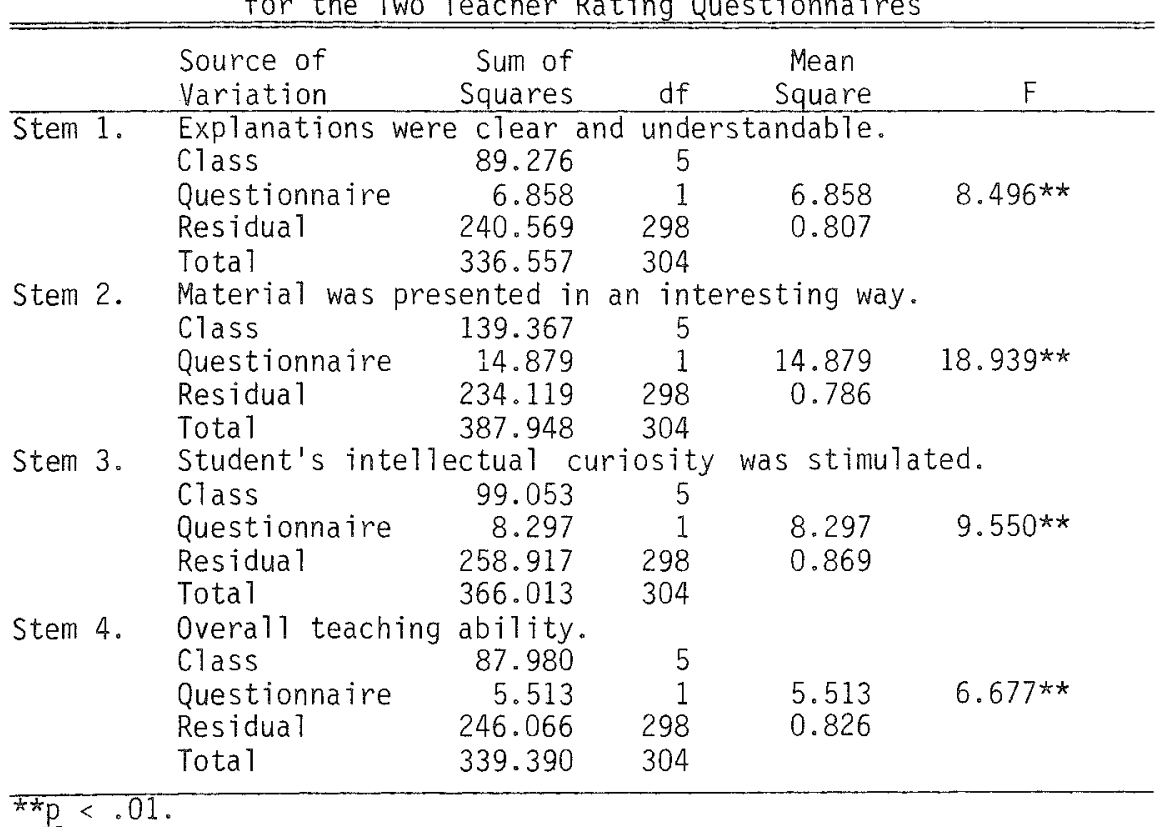

In addition to the means, the reliabilities (Table 4), calculated by Horst's (1949) generalized formula, and two other distributional parameters, the variance and the coefficient of skewness (Table 5), were examined. Although the variances for the two questionnaires did not differ significantly, the coefficients of skewness are larger for each item stem for $Q_{2}$ than $Q_{1}$. Reliabilities were high for both scales.

Finally, all of these effects are visually represented for Item 4, overall teaching effectiveness, in Figure 1. (Plots of the other items give nearly identical results.)

\section{Discussion}

Clearly, the degree of negative skew in distributions of behavioral rating measures can be altered by the verbal labels used as anchors. Both means and skewness coefficients were affected by the verbal labels used in this research. Data presented also show that a rating scale anchored by a 
Table 4

Item Reliabilities and Paired t-tests for Differences in the Mean Ratings on the Two Teacher Rating Questionnaires

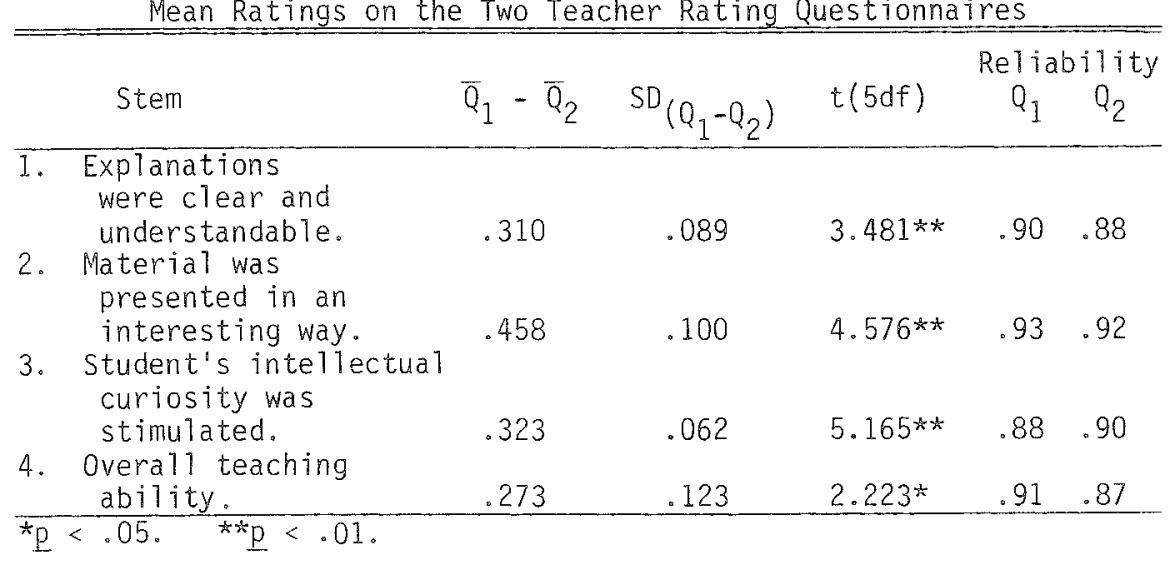

Table 5

Means, Variances, $F$ for Variances, Wilcoxon $W$, and Coefficients of Skewness and Kurtosis for Questionnaires 1 and 2

\begin{tabular}{|c|c|c|c|c|c|c|c|c|c|c|c|c|}
\hline & \multicolumn{2}{|c|}{$\mathbb{N}$} & \multicolumn{2}{|c|}{ Mean } & \multicolumn{2}{|c|}{ Variance } & \multirow[b]{2}{*}{$F$} & \multirow[b]{2}{*}{ W } & \multicolumn{2}{|c|}{ Skewness } & \multicolumn{2}{|c|}{ Kurtosis } \\
\hline class & $s_{1}$ & $Q_{2}$ & $\mathrm{Q}_{1}$ & $a_{2}$ & $Q_{1}$ & $Q_{2}$ & & & $Q_{1}$ & $Q_{2}$ & $\mathrm{Q}_{1}$ & $Q_{2}$ \\
\hline Item & 1: & Expl & nations & were & lear and & understa & ndable. & & & & & \\
\hline 1 & 25 & 25 & 3.560 & 3.120 & 1.090 & 1.277 & 1.172 & 1.556 & -0.650 & 0.312 & 0.140 & -0.670 \\
\hline 2 & 21 & 23 & 2.571 & 2.304 & 0.957 & 0.676 & 1.416 & 0.748 & 0.311 & -0.110 & 986 & -0.576 \\
\hline 3 & 33 & 32 & 1.848 & 1.594 & 0.945 & 0.572 & 1.652 & 1.038 & 1.407 & 0.855 & 2.369 & -0.673 \\
\hline 4 & 20 & 21 & 2.000 & 1.571 & 0.632 & 0.657 & 1.040 & 1.974 & 0.699 & 1.613 & 0.807 & 2.821 \\
\hline 5 & 26 & 25 & 2.462 & 1.920 & 0.658 & 0.910 & 1.383 & $2.088^{*}$ & 0.377 & 0.483 & $=0.206$ & -1.080 \\
\hline 6 & 27 & 27 & 2.074 & 2.148 & 0.533 & 0.823 & 1.561 & -0.253 & 0.525 & 0.356 & 0.733 & -0.565 \\
\hline Item & 2: & Mate & a] was & preser & ited in an & interes & ting way & & & & & \\
\hline 1 & 25 & 25 & 4.080 & 3.560 & 0.577 & 1.007 & 1.745 & 1.954 & -0.759 & -0.044 & 1.062 & -0.971 \\
\hline 2 & 21 & 23 & 2.762 & 2.130 & 0.890 & 0.664 & 1.340 & $2.136^{*}$ & 0.132 & 0.297 & 227 & 231 \\
\hline$?$ & 33 & 32 & 1.909 & 1.531 & 0.960 & 0.451 & $2.129 *$ & 1.537 & 1.040 & 0.903 & 0.302 & -0.243 \\
\hline 4 & 20 & 21 & 2.750 & 2.190 & 0.934 & 1.162 & 1.244 & $1.668^{*}$ & 0.559 & 0.378 & 0.176 & -1.088 \\
\hline 5 & 26 & 25 & 2.577 & 1.920 & 1.054 & 0.660 & 1.597 & $2.338 * \pi$ & 0.016 & 0.660 & -1.081 & 0.258 \\
\hline 6 & 27 & 27 & 2.037 & 2.037 & 0.499 & 0.729 & 1.461 & 0.112 & -0.052 & 0.326 & -0.854 & .636 \\
\hline I tem & 3: & Stuc & nt's in & tellect & ual curio & sity was & stimula & ated. & & & & \\
\hline 1 & 25 & 25 & 3.880 & 3.600 & 0.943 & 1.167 & 1.238 & 1.001 & -1.226 & -0.173 & 2.187 & -1.181 \\
\hline 2 & 21 & 23 & 2.619 & 2.261 & 0.648 & 0.747 & 1.153 & 1.539 & -0.428 & 0.365 & 0.055 & -0.219 \\
\hline 3 & 33 & 32 & 2.303 & 1.875 & 1.218 & 0.500 & $2.436 * *$ & 1.417 & 0.982 & 0.182 & 0.566 & -0.890 \\
\hline 4 & 20 & 21 & 2.650 & 2.476 & 1.082 & 0.762 & 1.420 & 0.485 & 0.491 & 0.329 & -0.031 & -0.409 \\
\hline 5 & 26 & 25 & 2.308 & 1.760 & 0.622 & 1.273 & 2.047 & $2.923^{* *}$ & +0.433 & 2.208 & 0.147 & 4.083 \\
\hline 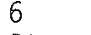 & 27 & 27 & 2.519 & 2.370 & 0.721 & 0.858 & 1.190 & 0.384 & 0.345 & -0.217 & -0.463 & -0.923 \\
\hline Item & 4: & Over & 11 teacl & hing ab & ility. & & & & & & & \\
\hline 1 & 25 & 25 & 3.720 & 3.320 & 1.043 & 1.060 & 1.016 & 1.504 & -0.655 & 0.280 & 0.607 & -0.964 \\
\hline 2 & 21 & 23 & 2.524 & 2.348 & 0.562 & 1.146 & 2.039 & 0.850 & 0.305 & 0.678 & -0.075 & 0.276 \\
\hline 3 & 33 & 32 & 2.061 & 1.656 & 0.966 & 0.491 & 1.967 & 1.576 & 0.676 & 0.600 & -0.477 & -0.71 .4 \\
\hline 4 & 20 & 21 & 2.400 & 1.952 & 0.674 & 0.648 & 1.040 & 1.585 & 0.355 & 0.090 & -0.065 & -1.417 \\
\hline 5 & 26 & 25 & 2.423 & 1.920 & 0.734 & 1.410 & 1.921 & $2.291^{\star}$ & 0.258 & 1.464 & -0.362 & 1.809 \\
\hline 6 & 27 & 27 & 2.074 & 2.370 & 0.379 & 0.704 & 1.858 & -1.423 & -0.036 & 0.021 & -0.094 & -0 \\
\hline
\end{tabular}

Downloaded from the Digital Conservancy at the University of Minnesota, http://purl.umn.edu/93227. May be reproduced with no cost by students and faculty for academic use. Non-academic reproduction requires payment of royalties through the Copyright Clearance Center, http://www.copyright.com/ 


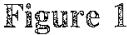

Comparison of Percentage Frequency Polygons for Questionnaires 1 and 2

on the Overall Teaching Effectiveness Item
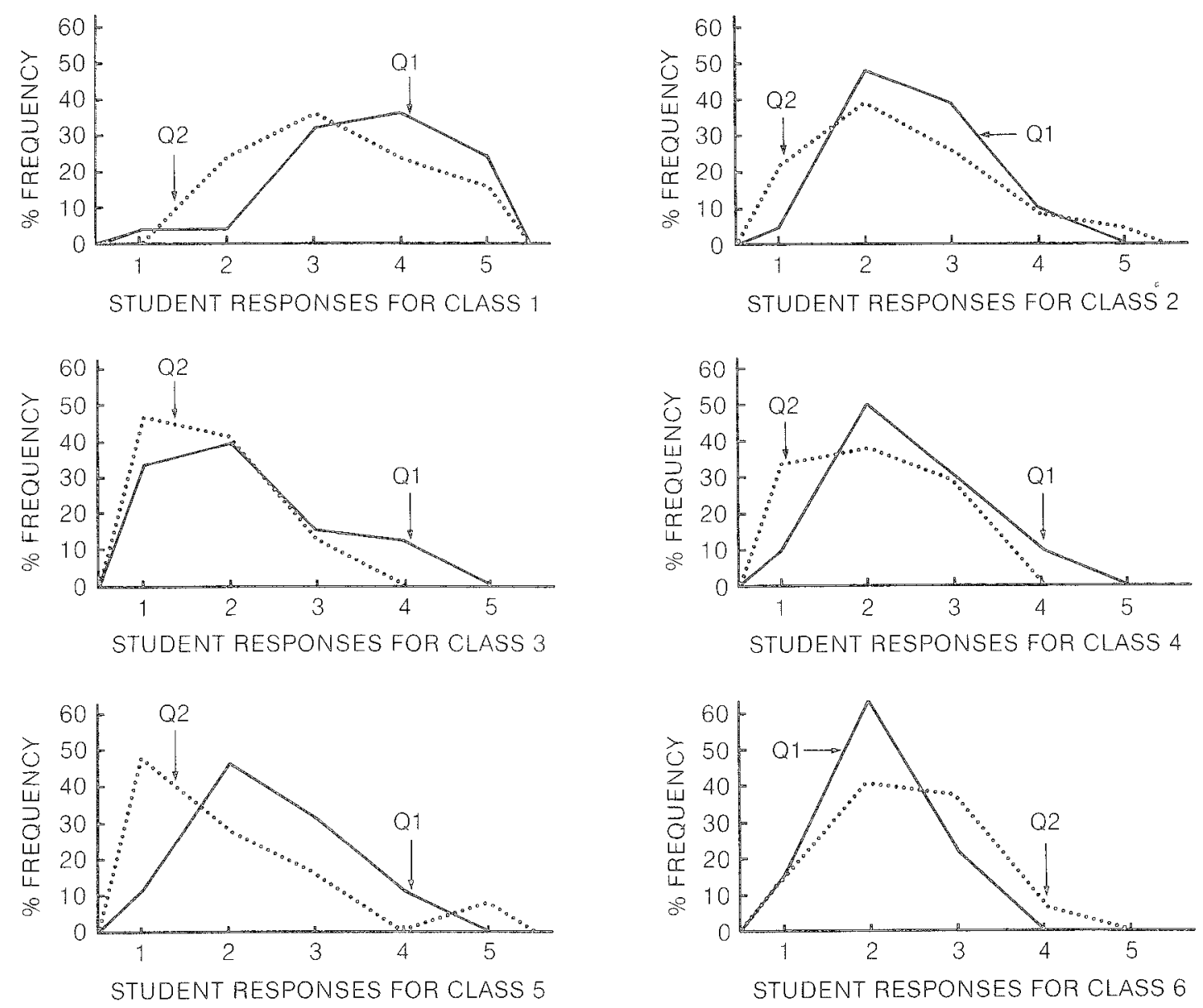

set of verbal labels more positive than those typically used (having higher descriptor indices) results in a shift of the mean numerical rating toward the less positive end of the scale; conversely, rating scales that are anchored by a set of less favorable labels result in mean numerical values shifted toward the favorable end of the scale. A finding of Lam and Klockars (1982) bears some similarity to this finding. Using entirely different methodology and investigating a different question about rating scales, their results indicate that the relationship between scales having all intermediate points la-

beled verbally and those having only the endpoints labeled is a function of the psychophysical scale values of the intermediate labels. Among the rating scales they used, lower means resulted for scales whose response categories were described by labels with more favorable psychophysical scaling values; ratings with verbal labels of less favorable scaling values produced higher means.

The relationship of the observed nonsignificant differences in variability for $Q_{1}$ and $Q_{2}$ to the leniency problem is not a simple one. To understand this, it must be remembered that there are two 
levels of variability in these ratings: first, there is variability among the students in a single class in their judgments of the teacher (i.e., $s_{\text {among judges }}^{2}$ ) and second, there is variability in the class means across teachers (i.e., $s_{\text {teacher means }}^{2}$ ). In order for ratings to have high reliability, the among-judges variability should be low, and the variance of mean ratings should be high, indicating ability to discriminate among those rated. That among-judges variability was not significantly different for $\mathrm{Q}_{1}$ and $\mathrm{Q}_{2}$ suggests that altering the verbal labels, at least for those in this research, did not result in greater disagreement among students within the same class in judging their teacher.

The number of teachers rated in this study is not large enough to addiress variability of mean ratings across teachers. However, the finding that mean ratings were affected by the verbal labels used strongly suggests that the failure to discriminate among various levels of good performance, which characterizes the leniency effect, would be lessened if labels were very carefully chosen.

Several findings that have implications for the construction of rating measures emerged from the calculation of descriptor indices. First, the use of the term "average" as a midpoint anchor is called into question, as well as its frequently associated labels "above average" and "below average." Although more rescarch is needed with ratings of other complex behaviors, students judging teaching behaviors characterize significantly less than $50 \%$ of their teachers by the term "average."

Second, an inspection of the descriptor indices reveals an important result - that adjectives whose meanings are logical opposites do not characterize numerically opposite proportions of a scale. Compare, for instance, the descriptor indices for a scale that uses what appear to be logically balanced adjectives, such as Very Effective (DI $=77$ ), Effective (58), Ineffective (19), and Very Ineffective (12). Although students characterize $23 \%$ of their teachers as falling at or above the label Very Effective, only $12 \%$ are characterized as falling below Very Ineffective. Similarly, $42 \%$ fall at or above Effective, only $19 \%$ at or below Ineffective. The same holds for other logically balanced de- scriptors, e.g., Very Good (79), Good (56), Poor (15), and Very Poor (11). The obvious lack of symmetry in the scale positions of these labels indicates that they are not at all the balanced sets they have been assumed to be if the scales are to represent the empirically determined range of performance. If the finding of adjective asymmetry extends to the judgment of other domains of human performance as well, then substantial questions arise about the results that have been obtained with scales employing such logically balanced anchors, including the use of polar adjectives to define scale endpoints.

From the point of view taken here, rating scale construction should begin by empirically determining the real range of performance in the behavior to be judged. This range can be ascertained by calculating a descriptor index for each of a set of possible verbal labels, just as test construction begins with the determination of item difficulty levels. Choice of labels on the basis of the obtained descriptor indices can then make possible the construction of scales that produce finer discriminations by raters within the portion of the response continuum of concern to the investigator.

These findings also have consequences for the interpretation of rating measures. Most often, mean ratings are compared to the numerical midpoint of their scale. If some of the common anchor descriptors have been used, then such a comparison could lead to severely distorted interpretation.

Although this research has shown some of the effects of verbal anchors on numerical ratings, there is no intention of implying that labels are the only determiners of rating distributions. The number of scale points, the number of verbal anchors, and the numerical values associated with each of the scale points are factors that may exert an influence on the distribution in some fashion.

In addition to casting doubt upon the "leniency effect" as the major determinant of negatively skewed rating distributions, these research findings suggest that whether rating measures represent the continuum of performance that occurs in the real world (as do achievement tests) or a continuum defined by logically balanced adjectives has non- 
trivial psychometric consequences that need further examination in other domains of human performance.

\section{娟eferenter}

French-Lazovik, G. (1974). Predictability of students' evaluations of college teachers from component ratings. Journal of Educational Psychology, 66, 373385.

Guilford, J. P. (1936), Psychometric methods. New York: McGraw-Hill.

Horst, P. (1949). A generalized expression for the reliability of measures. Psychometrika, 14, 21-32.

Lam, T. C. M., \& Klockars, A. J. (1982). Anchor point effects on the equivalence of questionnaire items. Joumal of Educational Measurements, 19, 317-322.

Landy, F. J., \& Farr, J. L. (1980). Performance rating. Psychological Bulletin, 87, 72-107.

Osgood, C. E. (1952). The nature and measurement of meaning. Psychological Bulletin, 49, 197-237.

Stephenson, W. (1953). The study of behavior: Q-technique and its methodology. Chicago: University of Chicago Press.
Thorndike, E. L., \& Lorge, $\mathbb{1}$. (1944). The teacher's word book of 30,000 words. New York: Teachers College, Columbia University.

Wherry, $\mathrm{R}, \mathbb{I} .(1950)$. Control of bias in rating: Survey of the literature (Tech. Rep. No. DA-49-0853 OSA 69). Washington DC: Department of the Army, Personnel Research Section.

\section{Ackshowledgmerits}

The authors thank Drs. Lloyd Bond, Charles A. Perfeti, and Mary $A$. Hartz for their critical reviews and valuable comments on this research. Special appreciation is expressed to Dr. Sara Strouss and Marian Skoog for help in preparing the manuscript and to Stephen Hennings for the preparation of Figure 1 .

\section{Adethor" Address}

Send requests for reprints or further information to Grace French-Lazovik, Director, Office for the Evaluation of Teaching, 3600 Cathedral of Learning, University of Pittsburgh, Pittsburgh PA 15260 , U.S.A. 